\title{
Parental Perceptions of Mental Health Disorders, and the Effect on Their Children
}

Rebecca Strange ${ }^{1 *}$

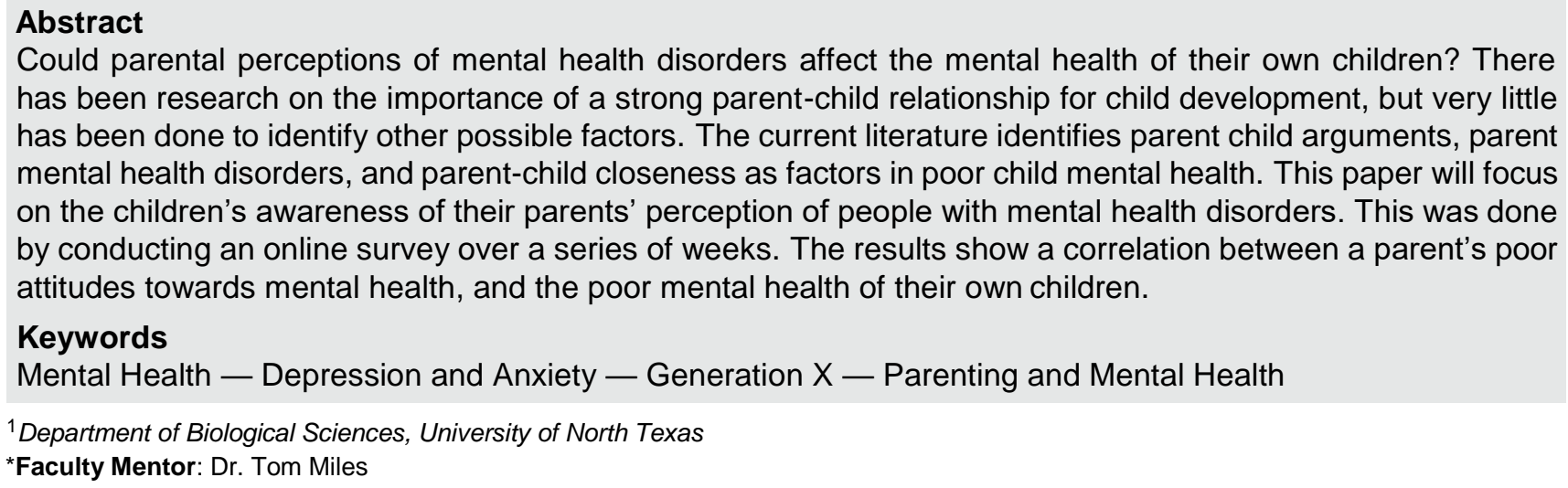

\begin{tabular}{lll} 
& \multicolumn{3}{c}{ Contents } \\
& Introduction & 1 \\
1 & Background & 1 \\
2 & Study Design & 2 \\
3 & Results & 3 \\
4 & Discussion & 4 \\
5 & Conclusion & 4 \\
& References & 4
\end{tabular}

\section{Introduction}

Depression and anxiety disorders are more common than ever in today's teenagers. The millennial and gen X generations have been referred to as the "Xanax generation", due to the overwhelming need for anxiety medication. While there has been research in to the importance of a strong parent child relationship when it comes to mental health, there has been little examination into what makes this relationship strong. In this paper, the effects of parental attitudes towards mental health disorders will be examined to see if there are any longlasting effects for their child's own mental health.

Growing evidence suggests that an adolescent's relationship with their parents correlates directly with their mental health. In one study, the number of disagreements with parents was directly associated with high symptoms of anxiety and depressive disorders. This study was conducted on 303 families, and consistently found that the level and number of arguments with parents throughout childhood had a lasting effect on the mental health of the children.[1] This data reinforces the theory that mental health disorders can be caused by a gradual buildup of stressful events and a gradual development of systems. This also suggests that parental influence has even more effect on adolescent mental health disorders than we previously thought. By arguing with their children, parents are inadvertently causing their children to develop poor coping mechanism for negative thoughts. The children are internalizing them and defining themselves by them. If these disagreements were about the negative thoughts themselves, would there be an even bigger consequence?

\section{Background}

It is common knowledge that teenagers can pick up traits from their parents. Unfortunately, according to 2014 study these traits can include anxiety, depressive, and eating disorders. Children of parents with a mental health disorder were significantly more likely to develop their own mental disorder. In a study conducted on 235 adolescents, parental emotional dysregulation was found to be correlated with adolescent emotional dysregulation.[2, 3] Parental eating disorders also significantly increased the chances of adolescent eating disorders, especially for adolescent girls. These findings are evidence that the ways parents cope with emotions is something that children can inherit, whether beneficial or not. This is supported by an American sociological review on children's inheritance of parental attitudes, which found that parental influence is significant in early childhood.[4] Although the influence may decrease with age, it is highly likely that children's perceptions on emotions and mental health are taught to them through their parent's actions. $[5,6]$ This could lead to a child learning that their negative emotions are wrong to feel and feeling guilt and confusion over them. This can also affect 
adolescent's willingness to confide in their parents, which is the only way for them to get access to professional help at a young age. Children are naturally less likely to confide in their parents in a topic they perceive their parents will disagree with them on.[7] If a parent's behavior has demonstrated that they are unwilling to sympathize with mental health disorders, children will not feel safe disclosing their own feelings and asking for help.

But what about parents who have experienced these feelings themselves? In a 2014 study on parental perceptions of their children's risk of developing anxiety and depression, the parents' perceptions were far from reality.[8] Most of the parents were concerned with the possibility but denied that their own mental health would have any effect on their children's risk of developing the same disorders. They did acknowledge that their anxiety and depression affected their family's quality of life but attempted to hide this from their children to "protect them". Most were unaware of the professional help available for their children, and many said parental help should be sufficient for the children. This data demonstrates that not even parents who suffer from the same mental health disorders as their children are guaranteed to have a positive attitude about it and are just as unlikely to seek help for their children. By not discussing their feelings to "protect" their children, they also decrease the chances of their child confiding in them. This supports the theory that a negative attitude about mental health can come from anyone, and that they influence children.

But would therapy and professional intervention help these children even if they felt comfortable enough to ask for it? According to a 2008 study, $80 \%$ of children with above average anxiety reported significant quality of life improvements after just twelve sessions of over-the-phone cognitive behavioral therapy.[9] The children with significant enough anxiety to qualify for sertraline, an anti-anxiety medication, reported similar improvements. Parental involvement in these treatments was found to increase the effectiveness even more. Unfortunately, as demonstrated by the 2014 study, parental involvement may be difficult to achieve.

\section{Study Design}

Due to expected lack of parental cooperation, my survey focused on the child's experience. I conducted a survey of young adults who identify as having one or more mental health disorder, with a focus on depression, anxiety, and disordered eating. This survey consisted of questions clarifying the disorder and exploring the attitudes of the parents from the experiences of their child. Although these experiences may not accurately depict the way the parent views, this should not affect the results since the study is focusing on how children were affected by the expressed views only.

The survey was posted on Reddit, a social media site, over the course of a week. It received 37 responses. The survey began with some demographic questions:
- What age are you?

- What gender do you identify as?

Participants were then asked to select any mental health disorders you identify as having. The options were:

- Anxiety

- Depression

- Disordered eating

- Bipolar disorder

- $\mathrm{ADD} / \mathrm{ADHD}$

- Obsessive-Compulsive disorder

- None

- Other (please specify)

Participants were then asked how strongly do these disorder(s) affect you on a daily basis? Answer options were from 1 to 10 , with 1 being not very strongly and 10 being very strongly. They were also asked of they were receiving professional treatment for any of these disorders. Then, participants were asked: Do you self-medicate for any of the disorders? If so, then with what?

Finally, two scaled questions looked at participant attitudes toward their parental relationships. They were:

Answer the following questions on a scale of one to five, with one being strongly disagree.

1. My parents would have been comfortable with me going to therapy during my childhood/adolescence if money was not an issue

2. My parents were/are comfortable discussing emotions with me

3. My parents were/are understanding of people with mental health disorders

4. I had a strong relationship with my parents during childhood/adolescence

I feel like my relationship with my parents affected my mental health:

1. Yes, in a negative way

2. No

3. Yes, in a positive way

4. Other (please specify) 


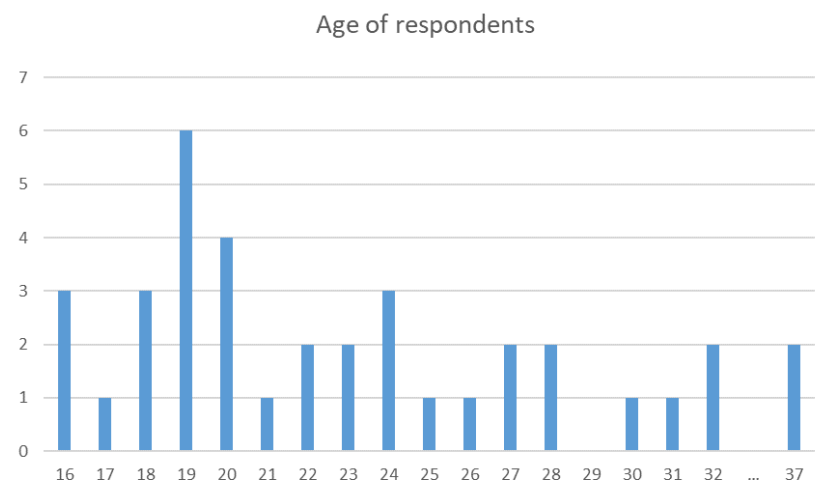

Figure 1. Range of Respondent Ages

\section{Results}

Respondents tended to be young adults, but ages varied. Respondent ages ranged from 16 to 37 with most being 18-20 years old. Results are shown in Figure 1. The respondents were mostly female $(n=25)$. Eleven respondents were male and one respondent identified as non-binary. As shown in Figure 2, the most common mental health disorders were anxiety and depression, which are the two that this study will focus on.

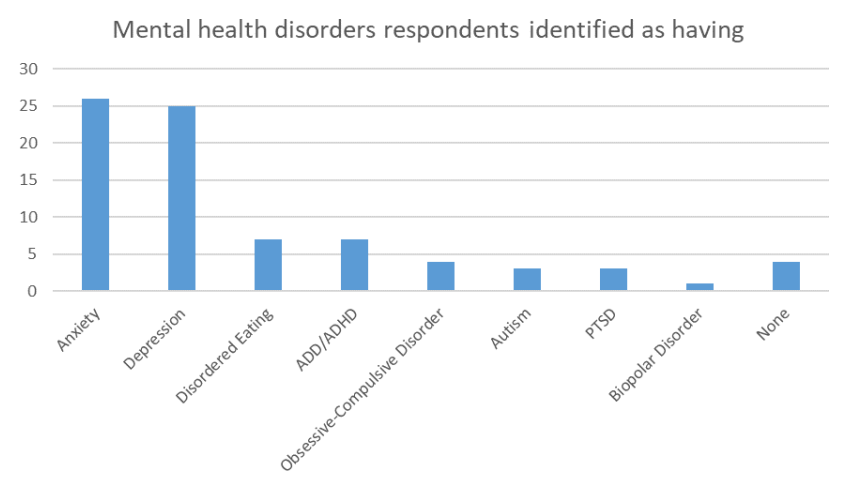

Figure 2. Distribution of Mental Health Disorders

As Figure 3 shows, the severity of the symptoms tended to be high. While four respondents reported no mental health disorders also reporting no severity of symptoms, the majority reported moderate to severe symptoms. At the top end of the scale, 24 reported symptoms between 8 and 10 on the 10-point scale.

There was no significant difference in the percentage of respondents with or without professional treatment. As many participants were under professional as were not. When asked if they were "self-medicating," 12 respondents (32\%) reported that they were using either substances like alcohol or cannabis, or compensative behaviors like yoga or meditation in order to control their symptoms. Figure 3 gives the breakdown: cannabis was the most popular substance used to self-medicate, with alcohol a second favorite.

A large majority of respondents felt that their relationship with their parents had a negative effect on their mental

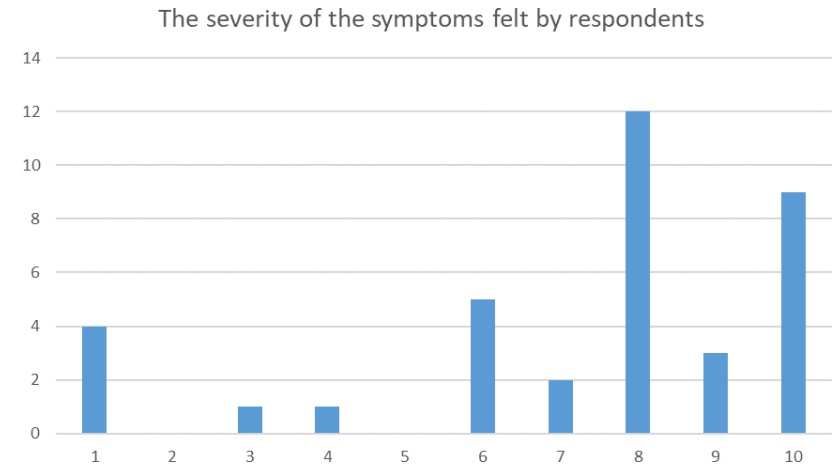

Figure 3. Severity of Mental Health Disorders

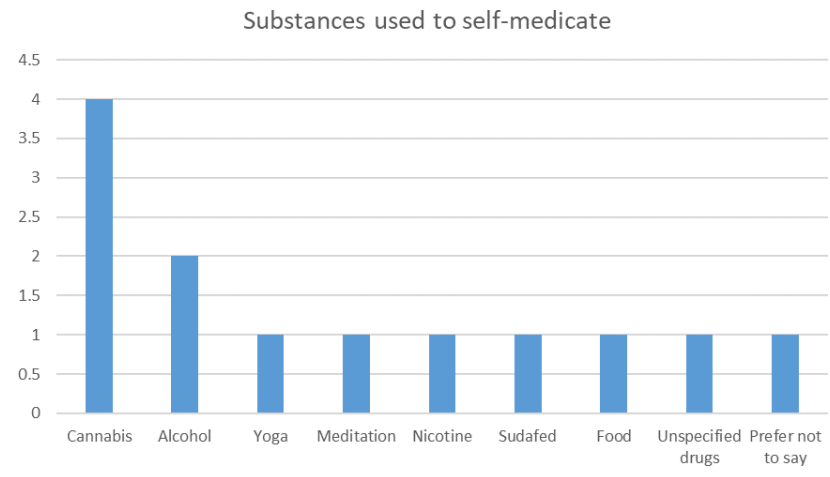

Figure 4. Self-Medicating Behaviors

health overall. Five respondents did respond with "other." The reasons given ranged from "not sure," "both positive and negative," and that there were differences between the relationship thy had with the mother and father. The "other" responses primarily reflect both a negative and positive effect of the relationship with their parents.

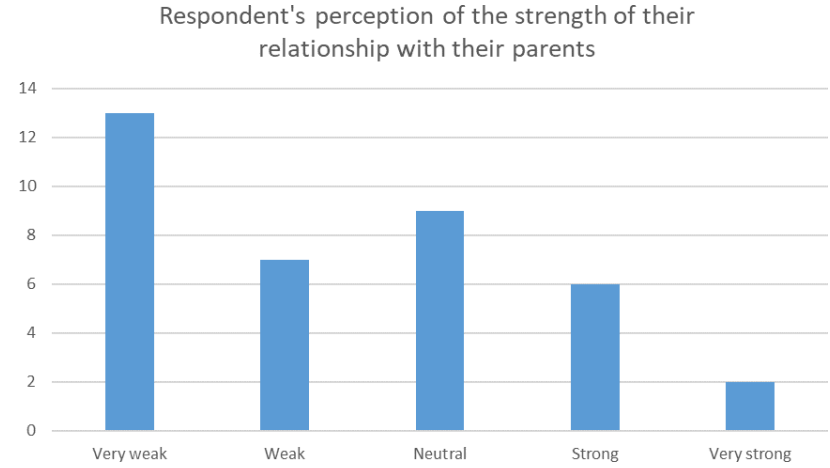

Figure 5. Strength of Parental Relationship

As shown in Figure 5, most respondents reported neutral to very weak relationships with their parents, with "very weak" being the most common answer. Further, most respondents felt their parents were neutral to very uncomfortable discussing emotions with them and most respondents felt their parents were neutral to unaccepting of people with mental health disorders. However, most respondents thought their par- 
ents would be neutral to willing to provide therapy, assuming there were no financial barriers.

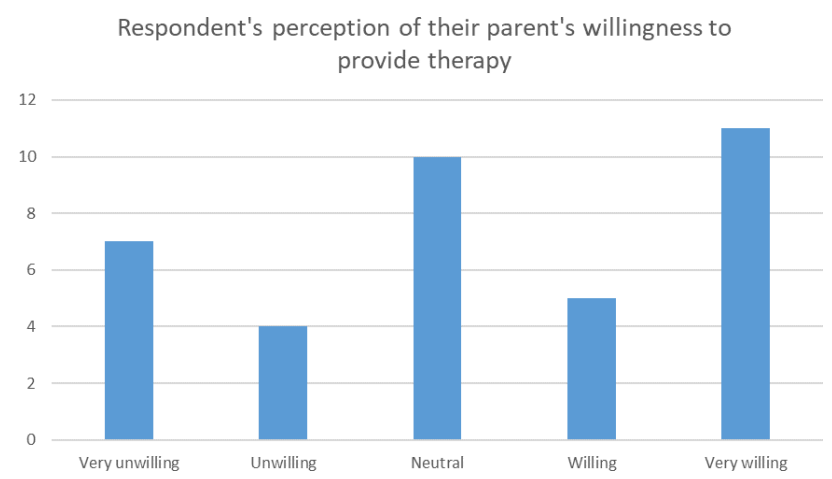

Figure 6. Willingness to Provide Mental Health Treatment

Overall, the results indicate that, although there is a willingness on the part of parents to provide treatment for mental health issues, there is a significant disconnect in ability to communicate. The majority of respondents report having weak relationships with their parents and feeling hesitation on their parents' parts to discussing mental health. As a result, many respondents turned to self-medicating behaviors as a means of coping with their anxiety and depressive disorders. These results have important implications as the amount and severity of mental health difficulties continue to increase.

\section{Discussion}

Overall, the respondents:

- Had moderate to severe symptoms.

- Felt their parents would be uncomfortable to neutral discussing emotions.

- Felt their parents would be neutral to comfortable sending them to therapy.

- Had poor to neutral relationships with their parents.

- Felt their relationship with their parents affected their mental health negatively.

There was no general consensus on if respondent's parents would be comfortable sending them to therapy. When patients were self-medicating, the majority reported using drugs or alcohol. These responses show a general societal trend of poor mental health, and of poor parent-child communication when it comes to mental health. $48.6 \%$ of respondents felt their parents were uncomfortable discussing emotions, and $48.6 \%$ felt that their parents were not understanding of people with mental health disorders. Only $21.6 \%$ of respondents reported a strong relationship with their parents.

On an individual level, respondents who did not think their parents were open to discussing emotions reported worse relationships with them, and were much more likely to report severe symptoms. There was no correlation between parent attitudes towards therapy and parent attitudes towards emotions and mental health disorders, which was unexpected. Since slightly over half of respondents reported seeking professional help, this means that parent attitudes have little effect on children seeking professional therapy.

\section{Conclusion}

There is a demonstrated trend between a parental unwillingness to discuss emotions and the mental health of their children. Children of parents who were unwilling to discuss mental health were more likely to say that their relationship with their parents affected their mental health and were more likely to say that they had a weak relationship with their parents. This study shows that this is an aspect of mental health disorders that should be explored further, especially as society trends towards open discussion of mental health. The effects of mental health being such a widely examined thing could mean that our next generation begins to break the trend of worsening mental health that we have seen for the past decades.

In the future, more in-depth studies would be needed to examine this link. One way to do this would be to simultaneously survey parents and their children and compare the parent's actual perceptions of mental health with the answers their children gave. This could be followed over the course of several years, to see if children with less understanding parents were more likely to develop strong symptoms of mental health disorders.

\section{References}

${ }^{[1]}$ Martha A Rueter, Laura Scaramella, Lora Ebert Wallace, and Rand D Conger. First onset of depressive or anxiety disorders predicted by the longitudinal course of internalizing symptoms and parent-adolescent disagreements. Archives of General Psychiatry, 56(8):726-732, 1999.

doi:10.1001/archpsyc.56.8.726

[2] Erika Hansson, Daiva Daukantaité, and Per Johnsson. Disordered eating and emotion dysregulation among adolescents and their parents. BMC psychology, 5(1):12, 2017. https://doi.org/10.1186/s40359-017-0180-5

[3] Susan L Warren, Lisa Huston, Byron Egeland, and L Alan Sroufe. Child and adolescent anxiety disorders and early attachment. Journal of the American Academy of Child \& Adolescent Psychiatry, 36(5):637-644, 1997. https://doi.org/10.1097/00004583-199705000-00014

[4] Jennifer Glass, Vern L Bengtson, and Charlotte Chorn Dunham. Attitude similarity in three-generation families: Socialization, status inheritance, or reciprocal influence? American Sociological Review, pages 685-698, 1986.

${ }^{[5]}$ Richard B Miller and Jennifer Glass. Parent-child attitude similarity across the life course. Journal of Marriage and the Family, pages 991-997, 1989.

${ }^{[6]}$ Lauren R Miller-Lewis, Alyssa CP Sawyer, Amelia K Searle, Murthy N Mittinty, Michael G Sawyer, and John 
Lynch. Student-teacher relationship trajectories and mental health problems in young children. $B M C$ psychology, 2(1):27, 2014.

https://doi.org/10.1186/s40359-014-0027-2

${ }^{[7]}$ Gene H Brody, Kris Moore, and Dana Glei. Family processes during adolescence as predictors of parent-young adult attitude similarity: A six-year longitudinal analysis. Family Relations, pages 369-373, 1994.

[8] Helma Festen, Karen Schipper, Sybolt O de Vries, Catrien G Reichart, Tineke A Abma, and Maaike H Nauta. Parents' perceptions on offspring risk and prevention of anxiety and depression: a qualitative study. BMC psychology, 2(1):17, 2014.

https://doi.org/10.1186/2050-7283-2-17

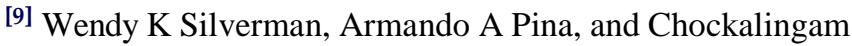
Viswesvaran. Evidence-based psychosocial treatments for phobic and anxiety disorders in children and adolescents. Journal of Clinical Child \& Adolescent Psychology, 37(1):105-130, 2008.

https://doi.org/10.1080/15374410701817907 